\title{
Exploration on New Tendency of Fur Clothing Design
}

\author{
Zhaofang Xu \\ School of Art and Design \\ Huanghe Science and Technology College \\ Zhengzhou, China 450006
}

\begin{abstract}
New concept of fur clothing becoming popularization has been put forward by discussing in several aspects such as the new tendency and trends of the international fur clothing market, progress of the fur design and new technology, the impact of modern life environment on the concept of life and consumption of the modern people, for predicting that the fur clothing of rabbit wool, cashmere and goose down will replace the high grade fur fashion represented by mink and fox skin, which becomes the new tendency of mainstream in the modern fur clothing design and fur clothing market.
\end{abstract}

Keywords-fur clothing; rabbit wool; cashmere; goose down; population; new tendency

\section{INTRODUCTION}

Fur refers to the animal skin which is tanned and processed for making clothes, used by the clothing designer and making for production of various costumes of refined style. The fur clothing can embody fully the status and wealth of its user by its magnificent appearance and outstanding craftsmanship. Therefore, the fur clothing is honored as the queen of garment materials, and fur has rich texture in outlook and magnificent. The visual and tactility feature, and its preciousness and rareness make it become the valuable thing of the noblewomen and debutantes.

There was record of "wearing fur in winter and wearing kudzu vine in summer" in "the five furs - Han Feizi". In Jin dynasty, the allusion of uninhibited Yuanfu "changing for wine with golden mink" has let us memorize the Mink. "flower-dappled horse and expensive mink, were taken out to trade for delicious wine", and the expensive mink has made the status and taste of fur clothing be changed to another kind of extreme while enjoying the mien of the poetic genius of one generation, making the concept of fur exceed its nature and become to be a kind of symbol of power and wealth. As early as in the empirical society, there is such a law, only people of special rights such as emperor family, noble group and monks can wear fur clothes, while concept of fur becomes increasingly narrow each day, becoming the special representing term for fine fur such as mink and fox skin.

Currently, the fur kind promoted to the world by the most authoritative fur organ -Saga Furs of Scandinavia and NAFA group has only two, mink and fox skin. The mink and fox skin have become the top kind due to their first grade quality and appearance, wining the favor by masters of top class. (They have pushed the fur clothing market into the Ivory Tower while pushing the design of fur clothing to the top of pyramid. Just as the crisis of high grade fashion in the 1970 's, the medium and lower grade fur clothing of rabbit wool, cashmere and goose down oriented for common people, had produced enormous impact on the high grade fur fashion represented by mink and fox skin. The cashmere, rabbit wool, goose down and other fur finished clothes have developed fast with the push of new concept, new technology and new consumption group, as an inevitable tendency that for them to become the mainstream of the fur clothing market.

\section{RABBIT WOOL, CASHMERE AND GOOSE DOWN WILL REPLACE THE FUR CLOTHING REPRESENTED BY MINK AND Fox SKIN to BECOME THE MAINSTREAM OF THE FUR Clothing MARKeT.}

The primitive people used bone needles to stitch animal skins, which were put on by them to show their strength and power, and they are produced and developed along with the producing and development of civilization of the human beings.

In the empirical society, high grade fur became the exclusive use product and symbol of identity of a few ruling people. In ancient time, both Chinese and foreign emperors once issued some limiting decree to make strict regulations for people in different social stratification in wearing what kind of fur.

During the flourishing period of fur clothing, one brand new high grade Citroen car price was as high as 0.2 million Francs, while a high grade fur clothe of famous brand was priced 0.38 million Francs, so that the shop needs to hire special security person to take care of it. In 1918, one exclusive shop specialized in designing fur coat for noblemen and Hollywood film stars appeared in Roma; in the 1930's, fox skin rush appeared, making fur become the necessary material for fashion. The film star of movie "Desire", Marin Dietrich's image and outstanding performance wearing expensive fox skin cappa made the popularity of fur reach the top point. Thereafter, the joining of some international masters and image of film stars for fur clothing, have pushed the fur industry to an era of extreme 
brilliance, and fur clothing has become the valuable staff for people wearing and collection thanking to its ventilation, moist absorption, warmth, durability and luxury.

However, the fur resistance activity has had a history of more than a decade, and extremist of environmental protection may even attack the human body of fur cloth wearing person directly, which is the main reason why the international fur clothing market has been down in recent years. People start to care for the rights of animal. The crisis of high grade fashion industry happening in 1960's and 1970 's, affected the high grade fur clothing industry, plus the extremism act of the environmental protection persons, the fur clothing industry decaying was accelerated. The status symboling the identity in the past started to be moved, which pushes forward the process of fur clothing from customization to finished product. The unbeatable high grade fur industry has become to be ending over, and the concept of clothing design including fur cloth has changed greatly. For example, by using high grade fur clothing design and craftsmanship, cheap fur clothing market is developed in even larger market, such as cashmere, rabbit wool, goose down and other common fur products.

\section{The OBJECTIVE CONDITION AND ADVANTAGES FOR RABBIT WOOL, CASHMERE AND GOOSE DOWN TO BECOME THE MAINSTREAM OF THE FUR MARKET}

The international fur market is a very active market with large annual trade volume and large price fluctuation. Though artificial fur product appeared recently for replacement product, its performance and appearance can not compete with the natural fur at all. Since the second half of 1987, production of fox skin and mink was larger than the sale, due to fur industry affected by the western economic crisis and fur resistance activity. At that time, high grade fur clothing market is not good, but the medium and lower grade fur market was still active, in addition that the designers continued to provide ever-changing style, which having broken the quiet situation at the time. In the Shanghai China senior fashion show, the 30th China international fur exchange fair, APIE Asia-pacific fur display fair, German Dusseldorf fashion show and other several exchange fairs, many big foreign businessmen who managing senior fur product such as mink and fox skin, have shown extremely large interests in rabbit wool, cashmere and goose down, and they said the mink and fox skin business becomes harder and harder to do recently, and rabbit wool, cashmere and goose down and other low price product start to attract attentions of people in the market. Some people predicted the situation the senior product commands the fashion industry in fur market will be broken soon.

\section{A. Impact of Social and Economic Environmental Factors}

The fashion industry starts to challenge the traditional aesthetics of clothing, and designers release their calls for giving a little more green to the earth and giving a little more warm feeling to the human being by their products, to make the strange feeling and distance feeling between people be minimized, and have a little more intimation to the nature and natural environment.
The 21st century is a time of deflation, which has a large effect on people's demands for one of the senior consumables, fur products. Just like in the situation in the 1970's for senior fashion, the consumption group shrank fast, and lots of senior fur product company faced severe crisis, which also promoted development and exploitation of some medium and lower grade fur such as rabbit wool, cashmere and goose down.

\section{B. Air Temperature and Environmental Factor}

According to the record of climate, the average ground air temperature of the globe appears in obvious rising tendency, and till 2050, the range of global warming may be between 4.15 and $10^{\circ} \mathrm{C}$. The change of external living environment makes the fur clothing change the unreachable noble image of being thick, heavy and big in the past and develop toward the fashion concept of being light, thin and soft. For mink and fox skin and other fine fur, the thick and heavy sense can not be changed largely due to the structural of the wool and plate of itself. The global warming makes it possible for fur to develop in direction of being light, thin, soft and beautiful, while the goose down, rabbit wool and cashmere are the kinds conforming to the condition of being light, thin, soft and beautiful most. The life of modern people has close relation with the air temperature change, and rabbit wool, cashmere and goose down are not confined by it, being the best material for making fur clothing.

\section{THE SUBJECTIVE CONDITION AND AdVANTAGE FOR RABBIT WOOL, CASHMERE AND GOOSE DOWN BECOMING THE MAINSTREAM OF MARKET}

Cost for raising rabbits, sheep and goose is low and their reproduction is fast, thus ensuring the market supply at low price. The market price of goose down is RMB 30 to 40 per piece of fur, and the cost price of rabbit fur transported to China from Belgium, Spain and other western European countries is also only 0.13 to 0.15 US dollar per piece of fur. The product manufactured from them has a feature with small investment, fast profiting and high additional value in terms of design, production, management and so on. These unique advantage and property have attracted attentions from people at home at abroad, having good development prospective in international market. In recent years, China has strengthened cooperation with the first class brand company in the world and between its designers, and Shijia Fur Clothing Company opened a design center in Beijing too, providing the latest international fashion information for the fur manufacturing industry and designers in China. Goose down has also the feature of strong fur plate, white wool like snow, and so on, and its wool depilation resistance and feature of being fluffy are better than mink, its thermal performance is as good as fox fur, so it can be used to make various types of clothing.

\section{A. The Nitrate Dye Technology for Mink and Fox Fur Can Also Be Applied to the Handling and Making of Cashmere, Rabbit Wool and Goose Down}

The nitrate dye technology pushes the natural products into the world with tendency of popular fashion and brand - 
new appearance. The advanced technology and various rich chemical fuels make the designers have more options, for satisfying the requirements of stratification of people, and ensuring the consumption concept that fur clothing is multiplied, for civilian and common people. In recent years, China has made great progress and development in nitrate dye technology for fur making, ensuring the rabbit wool, cashmere and goose down to be in line with the global popularity requirement in terms of raw material processing of rabbit wool, cashmere and goose down, which is as follows:

1) Treatment of the wool appearance. Bleaching: aiming to whitening the wool.

Bleaching dye: divided into highlight dye, gradual dye, single color dye, multiple color dye and local dye.

Spray dyeing: the popular one is the iridescence, the phantom, the frost on grass, one point and two colors, even one point and three colors, spray ridge sub of chinchilla imitation and so on.

Printing: printing dye only has effect on the surface of the fur, without penetrating to the bottom of fur and the plate. Such a process is used mostly in rabbit wool bedding.

2) Texture treatment. Different kind of fur has different condition and effect. It is exactly these different textures that make the unique feature of fur. The designers make selection from their different character to bring their individual feature into play fully by using their advantages. The new technological method gives fur brand new appearance to increase its 3D and sense of depth. Some methods used originally in wool pulling, cutting and flower cutting for mink and fox fur, have been fully applied in processing of rabbit wool, cashmere and goose down. Printing will be done after wool cutting is done on the fur surface, which appears in art effect of comparison and combination of plane and 3D, printing and net color, thus not only the appearing effect being able to show various pattern according to the creative idea of designers, but also compensating the rabbit wool being bold to improve its use rate greatly. Besides, it is very suitable to large scale production, and the beauty, low price and high efficiency have made them have the best condition for becoming the mainstream of the fur clothing industry.

3) Fur plate treatment. Today, people have higher and higher requirement for the fur product being light, thin, soft and warm. To achieve such effects, the beautifying of fur plate has become very important while making various technological treatments for the wool. Along with the progress and development of nitrate dye technology, the fur plate treatment has become more mature. The modern nitrate dye process has made the fur plate to show in the brand new appearance, which was beautified with the lining, by processing of the plate and rich method. In the continuous innovation of fur technology, the fur clothing has formed a new concept different from that in the past, which conforms to the requirements of people for its being light, thin, soft and warm to a bigger degree.

4) Greening of fur. As people pay greater attention to the environmental protection, returning to the nature, protecting the earth, protecting the environment, using of big amount of natural material product and environmental protection material, are the two aspects of greening consumption tendency. And more attention is paid to the latter than the former, which is shown mainly in: love for various natural materials such as cotton, hemp, silk, wool and animal byproducts; the strict control and even prohibition of the harmful composition in various dyes. Adapting to such a trend will be appreciated by the society, the tanning and nitrate dying process of rabbit wool, cashmere, goose down can completely meet the requirements of environmental protection of greening, thus satisfying the people's needs and achieving the goal of the protection of the environment at the same time.

\section{CONCLUSION}

In the fashion stage of the new century, the fur clothing will be so common that common people can possess it, which can adapt the multiple consumption requirements of different consumption stratifications. Meanwhile, the imitation and following of trends are very common, so it is the popular peak phase and a period of the largest sales volume of enterprises. Rabbit wool, cashmere and goose down can satisfy the requirements of wide consumers in quantities, and its low cost is more suitable for market sales in batch, and the comprehensive art processing of the wool and fur plate, will make it be more in line with the requirements of fashion and vogue, and become a sign for youth and fashion.

Along with the continuous development of science and technology, and the continuous appearing of new material, new technology and new process, and under the effect of many other factors, people start to develop and use medium and lower grade fur (rabbit wool, cashmere and goose down) and make them into senior fur in imitation. Subject to effect of idea of liberty, democracy and equality, the popularity of vogue is not only a transmission from top to bottom, but also a transmission form bottom to top or a parallel transmission. The fur clothing unreachable in the past starts to enter the cabinet of common people too. The Fur garment for civilian, vogue and popularization is the inevitable trend. Rabbit wool, cashmere and goose down will also replace the fur clothing represented by fox skin and mink to become the mainstream of the fur market.

\section{REFERENCES}

[1] Ning Jun, Chen Guiling, Li Shuzhen, Lu Yaxin. "Development strategy for Beijing apparel industry" Journal of textile research, Sep. 2005. 